\title{
Study on the Mechanism of the Regulation of NAA Promoted the Adventitious Root Formation of the Dalbergia Odorifera.T.Chen Hardwood Cuttings
}

\author{
Hongxin Wang ${ }^{1, a}$ Yuan $\mathrm{Li}^{1, \mathrm{~b}}$ Li Zhang $^{1, \mathrm{c}}$ Bo Chen ${ }^{1, \mathrm{~d}}$ \\ ${ }^{1}$ Sanya University, Sanya, Hainan, China, 572000 \\ aemail, ${ }^{b}$ email, ${ }^{\mathrm{c} e m a i l,}{ }^{\mathrm{d}}$ email,
}

Keywords: NAA, Dalbergia odorifera.T.Chen, Hardwood Cutting Seedlings; Associated Enzymes; Rooting Characteristics

\begin{abstract}
In order to reveal the rooting mechanism of Dalbergia odorifera.T.Chen, the rooting mechanism of Dendrocalamopsis mongolica was 4,600 mg / L NAA rooting (POD), polyphenol oxidase (PPO), superoxide dismutase (SOD), indole (SOD), indoxylate (SOD), indoxidase Acetic acid oxidase (IAAO) and other root-related enzyme activity changes. The results showed that the rooting type of callus was decreased by $600 \mathrm{mg} / \mathrm{L}$ NAA, and the rooting rate and rooting quality were obviously improved. The activity of PPO, SOD and IAAO increased gradually after cutting, reached the peak at the time of root formation, and then decreased gradually. The activity of PPO, SOD and IAAO increased gradually after cutting. The activity of POD enzyme increased gradually after cutting, reached the peak at the root formation stage, then gradually decreased, then gradually increased, reached another peak at the root elongation, and finally decreased gradually, showing a bimodal trend.
\end{abstract}

\section{Introduction}

(Dalbergia odorifera.T.Chen) Another name is yellow pear, Papilaeaceae (Papilionaceae) Dalbergia (Dalbergia) trees, Hainan Province is unique rare and endangered species, for the national secondary protected plants [1], Is the manufacture of high-grade furniture and exquisite handicraft materials. Decoction of red sandalwood is resistant to drought and barren, adaptable characteristics, is worth promoting the cultivation of precious species [2]. The traditional way of breeding is to use sowing and breeding, long-term production practice shows that planting and breeding instability, the emergence rate is missing, and the use of cutting propagation method can effectively solve these problems. In this study, we investigated the effects of different concentrations of NAA on rooting of hardwood cuttings, and analyzed the effects of different concentrations of NAA on the effects of different concentrations of NAA on the rooting of shoots, (PPO), superoxide dismutase (SOD) and indole acetic acid oxidase (IAAO) were used to determine the establishment of high efficiency rhubarb cuttings, Tan tree species to promote the provision of scientific theoretical basis.

\section{The Test Ground}

In the Sanya College garden teaching test nursery to test. The site is located in Jianyang City, Hainan Province, located at $109^{\circ} 53^{\prime} 92$ "E, $18^{\circ} 32^{\prime} 72 "$ N, $200 \mathrm{~m}$ above sea level. The average annual temperature of $25.4{ }^{\circ} \mathrm{C}$, July the average maximum temperature of $28.3{ }^{\circ} \mathrm{C}$, the average monthly minimum $20.7{ }^{\circ} \mathrm{C}$ temperature, strong ultraviolet light, adequate light, the annual sunshine time of about $2563 \mathrm{~h}$. Dry and wet season is clear, 7 to 11 months for the rainy season, precipitation accounted for $85 \%$ of the year.

\section{Materials and Methods}

The Cutting Test. In early April 2016 in Sanya College nursery nursery cutting test, cut from the Sanya College Yataishan pear plantation in 2012 planting the fragrant red sandalwood base of the base of the year buds grow robust, no pests and lignification of hard branch. Remove the lower 
leaves, keep 2 to 3 upper leaves and shoots, cut into $8 \sim 10 \mathrm{~cm}$ length of the cuttings, the lower incision flat cut, with paraffin smear on the incision, cut a good cut every 20 for a bundle, 1000 times more carbendazim solution soak for $20 \mathrm{~min}$ after drying. And then immersed in the concentration of 400,600,800,1000,1200mg / L NAA solution soak $2 \mathrm{~h}$, the control was soaked with distilled water $2 \mathrm{~h}$. The cutting substrate is a mixed matrix of perlite, vermiculite, and fine river sand (volume ratio of 5: 3: 2). After cutting to maintain the shading rate of about $50 \%$, relative humidity control in $70 \%$ to $80 \%$, temperature control at $20 \sim 30{ }^{\circ} \mathrm{C}$, according to the need for spray treatment, conventional management. Completely randomized block design, each dealing with 80 cuttings, 3 replicates. New leaves fully expanded every $15 \mathrm{~d}$ after spraying 2 \%o urea.

Determination of Indicators. The callus formation and the adventitious roots were observed after cutting for 10 days. After every $2 \sim 3$ d observed 1 times, to the wound healing. (D), rooting initiation (d), rooting rate (\%), adventitious root number (bar), adventitious root length (cm).

Associated enzyme index. The results showed that POD, PPO, SOD and IAAO were measured at $3 \mathrm{~cm}$ of the basal part of the cuttings, and then the rootings were taken and the rootings were observed. Activity, 3 times repeated determination.

Determination Method. PPO activity was measured by pyrogallol colorimetric method [4]. POD activity was measured by guaiacol method [5]. SOD activity was determined by nitro-nitro-blue tetrazolium chloride method [5], IAAO activity was measured by colorimetric Method determination [6]. PPO was used as a viable unit (U) for $0.01 \mathrm{ml}$ units per g of fresh protein per minute of A410 optical density. POD was changed to 0.01 per 1 for 0.0170 per unit of peroxidase activity unit (U) SOD was used to inhibit the oxidative reduction of NBT by $50 \%$ of the enzyme activity of an enzyme activity unit (U), IAAO per $g$ fresh sample within $1 \mathrm{~h}$ decomposition of the destruction of IAA mg expressed 1 enzyme activity unit (U).

Data Processing. The data were analyzed and analyzed by Excel 2007 software. The variance analysis and Duncan's multiple comparison were performed by SPSS 18.0 software.

\section{Results and Analysis}

Effects of NAA Concentration on Rooting Characteristics of Hardwood Cuttings. The results showed that the cuttings of the incision and above $3 \sim 5 \mathrm{~cm}$ were observed after $10 \sim 20 \mathrm{~d}$ cutting (20 $30 \mathrm{~d}$ in the control group) after cutting with NAA. And with more protrusions; $20 \sim 30 \mathrm{~d}$ (control group for $30 \sim 40 \mathrm{~d}$ ) when there are some cuttings incision callus has been out of the obvious adventitious root tip, gradually forming the root system, adventitious root was significantly elongated.

Table 1 Different NAA treatment of red sandalwood hardwood cutting rooting situation

\begin{tabular}{|c|c|c|c|c|c|}
\hline $\begin{array}{l}\text { NAA } \\
\text { treatment } \\
(\mathrm{mg} / \mathrm{L})\end{array}$ & Callus occurs (d) & Take root (d) & Rooting rate \% & $\begin{array}{l}\text { Number of } \\
\text { indefinite root } \\
\text { (article) }\end{array}$ & $\begin{array}{l}\text { Distant root length } \\
(\mathrm{cm})\end{array}$ \\
\hline $\mathrm{CK}$ & $25.00 \pm 1.78 a$ & $33.83 \pm 1.47 \mathrm{a}$ & $38.34 \pm 1.72 d$ & $25.17 \pm 2.32 d$ & $3.17 \pm 0.2 \mathrm{~d}$ \\
\hline 400 & $24.18 \pm 1.77 b$ & $29.50 \pm 0.95 b$ & $40.23 \pm 1.58 c$ & $33.10 \pm 2.58 \mathrm{c}$ & $3.43 \pm 0.21 b$ \\
\hline 600 & $21.25 \pm 1.58 \mathrm{~d}$ & $24.35 \pm 2.01 \mathrm{e}$ & $52.14 \pm 1.99 \mathrm{a}$ & $42.35 \pm 2.31 \mathrm{a}$ & $4.35 \pm 0.52 \mathrm{a}$ \\
\hline 800 & $23.50 \pm 2.01 b c$ & $25.38 \pm 1.42 d$ & $42.31 \pm 2.58 b$ & $40.32 \pm 2.85 b$ & $4.07 \pm 0.22 c$ \\
\hline 1200 & $24.32 \pm 1.54 b$ & $26.35 \pm 1.61 c$ & $42.01 \pm 2.11 b$ & $39.15 \pm 2.85 b$ & $3.86 \pm 0.18 c$ \\
\hline
\end{tabular}

Note: The same data after the lowercase letters showed significant difference $(\mathrm{P}<0.05)$.

It can be seen from Table 1 that the callus of each treatment appeared $20 \sim 25 \mathrm{~d}$ after insertion, and the adventitious roots appeared on $24 \sim 29$ days, while the control showed callus at 25 and 33 days after cutting. All treatments of exogenous auxin NAA could shorten the callus emergence and adventitious root generation time. The rooting rates of the treatments were significantly higher than those of the control, and the rooting rates of the treatments were $4.93 \%, 35.99 \%, 10.35 \%$ and $9.57 \%$, 
respectively, in the number of adventitious roots and adventitious root length. In terms of rooting quality, the number and length of adventitious roots treated with $600 \mathrm{mg} / \mathrm{L}$ NAA were the highest in all treatments. In general, NAA treatment could improve the rooting rate and rooting quality of the hardwood cuttings of the red sandalwood, and $600 \mathrm{mg} / \mathrm{L}$ NAA had the highest rooting rate and the rooting quality was the best.

Changes of Related Enzyme Activities During Rooting of Hardwood Cuttings. POD is a highly active enzyme, can participate in a variety of physiological processes in the plant and the formation of lignin, and adventitious root induction and elongation growth is closely related to oxidize IAA, to eliminate the body too much endogenous IAA, with some higher plants The development process is closely related. The activity of POD is closely related to the rooting of in vitro plants, which is one of the landmarks of plant rooting [7]. In the adventitious root induction and expression period, the increase of POD activity is a sign of rooting ability [8]. It can be seen from Fig. 1 that the activity of POD in the cuttings treated with three different concentrations of NAA solution increased from 0 to 15 days after cutting, and reached the highest at 15 days and then decreased at 30 days Stage, again elevated, and then fell again. The results showed that $600 \mathrm{mg} / \mathrm{L}$ NAA could improve the POD activity of the cuttings, while the POD activity of the other four cuttings was the same as that of the control $(\mathrm{P}<0.05)$. The POD activity of the cuttings was $5 \sim 40 \mathrm{~d}$ and the $600 \mathrm{mg} / \mathrm{L}$ NAA treatment was significantly different from that of the control. The active state of POD is beneficial to the rooting of the cuttings. The activity of POD in all treatments was increased first and then decreased, then increased and then decreased, and the POD content in the cuttings of 400,600,1000,1200mg / L NAA was higher than that in the 15th day respectively, decreased by 15.32\%, 28.29\%, 38.63\%, 19.44\%, 19.73\%.

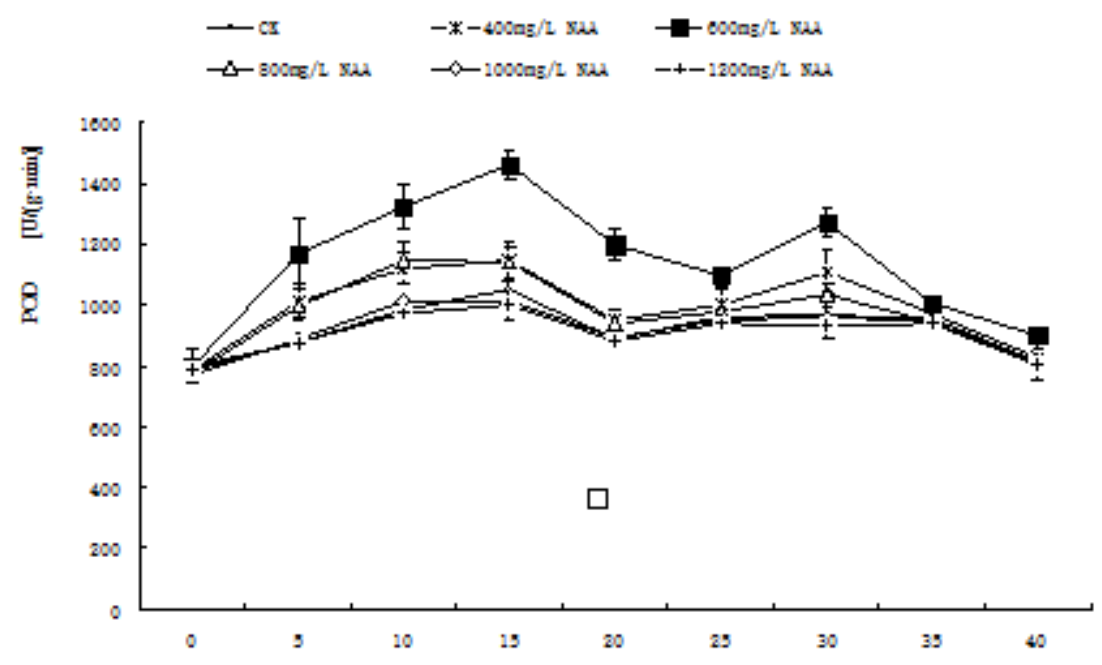

Fig.1 Changes of POD activity during hardwood cutting

PPO is a kind of copper-containing enzyme, which can catalyze the condensation of indole acetic acid (IAA) with phenolic substance "IAA-phenolic acid complex", which can promote the rooting factor of adventitious root formation and play an important role in plant rooting. But also with plant photosynthesis, resistance to pests and diseases, etc. [9-10]. It can be seen from Fig. 2 that the PPO activity of the cuttings of the control treatment increased gradually from 0 to 30 days, reached the peak at 30 days, and then decreased gradually, while the PPO activity of 400,600, 1000,12000 mg / L NAA In the $0 \sim 25 \mathrm{~d}$ are gradually increased, reached the peak at $25 \mathrm{~d}$, and then gradually decreased. The activity of PPO in $800 \mathrm{mg} / \mathrm{L}$ NAA treatment increased gradually from 0 to 15 days, decreased at 20 days, reached the peak at 25 days, and then decreased gradually. The PPO activity of $600 \mathrm{mg} /$ L NAA was significantly higher than that of control $(\mathrm{P}<0.05)$ within $5 \sim 40$ days. 


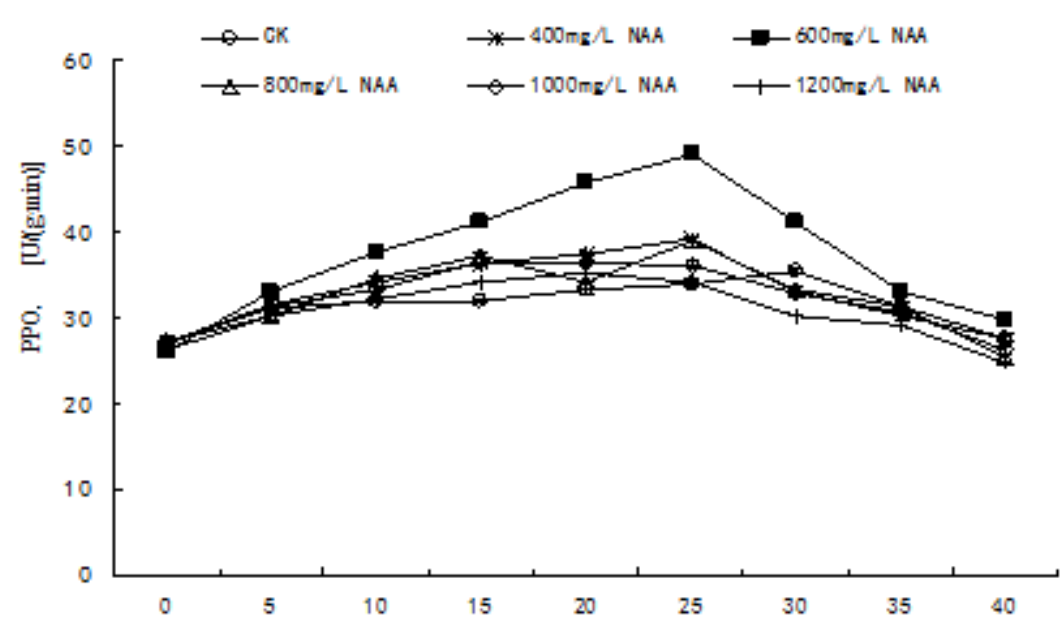

Fig.2 Changes of PPO activity during hardwood cutting

SOD (Superoxide Dismutase) is an effective scavenger of natural superoxide anion radicals in the body. It is a key enzyme for the metabolism of plants. It can catalyze the conversion of $\mathrm{O} 2$ to $\mathrm{H} 2 \mathrm{O} 2$ in vivo to reduce the toxic effects of free radicals on plants. Effectively inhibit the damage of free radicals to organisms, so that the plant to a certain extent, patience or resistance to stress (10). SOD activity affects the plant development process, and plant rooting is closely related. It can be seen from Fig. 3 that the SOD activity of the phloem in the cutting process of the different treatments was decreased first and then decreased. The activity of SOD in the cuttings treated with 400, 600 and $800 \mathrm{mg} / \mathrm{L}$ NAA increased gradually at the time of 0 to 25 days, reached the peak at 25 days, while the SOD activity at 1000 and $120 \mathrm{mg} / \mathrm{L}$ NAA was And reached the peak at $30 \mathrm{~d}$, then SOD activity decreased gradually. The SOD activity of the cuttings at different stages was significantly different from that of the control $(\mathrm{P}<0.05)$ except that $800 \mathrm{mg} / \mathrm{L}$ NAA was used for 10 days.

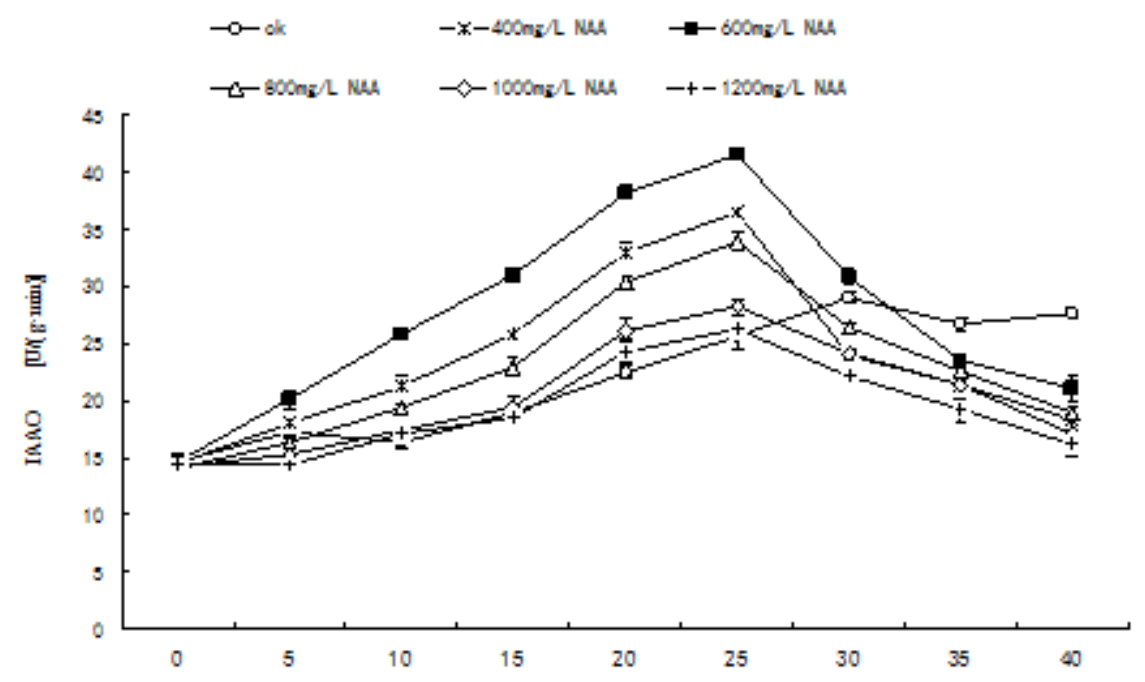

Fig.3 Changes of SOD activity during hardwood cutting

IAA important physiological function is to promote the formation of adventitious roots, its content changes will affect the advent of adventitious roots. IAAO can oxidize IAA, so the size of IAAO activity is also related to the occurrence of root [11]. It can be seen from Fig. 4 that the IAAO activity of all treatments increased first and then decreased in the process of hardwood cutting. The results showed that the activity of IAAO at 400, 600, 800, 1000 and $12000 \mathrm{mg} / \mathrm{L}$ NAA reached the highest level at 25 days, while the control treatment reached the highest level on the 30th day. The IAAO activity of the cuttings at each stage was significantly different from that of the control $(\mathrm{P}<0.05)$ except for $400 \mathrm{mg} / \mathrm{L}$ NAA treatment at day 5. 


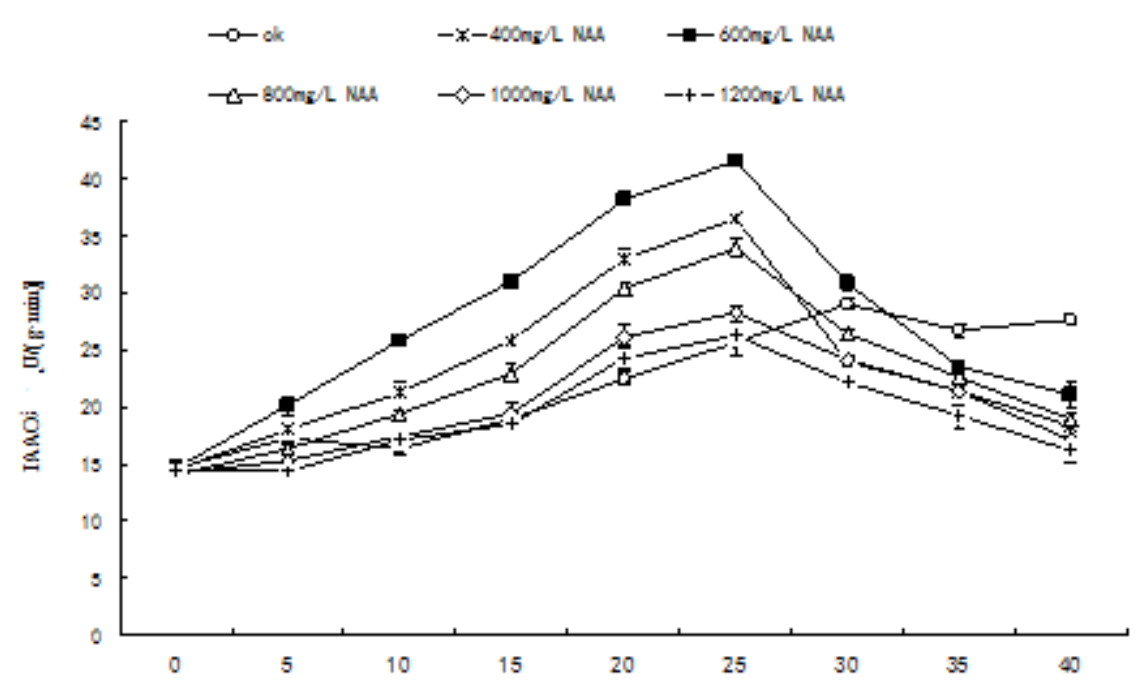

Fig 4.The Changes of IAAO activity during hardwood cutting

\section{Conclusion and Discussion}

Select the application of plant-promoting agents is to improve the survival rate of cuttings of trees one of the key technologies [12]. The results showed that the suitable concentration of NAA could significantly shorten the rooting of the hardwood cuttings of the red sandalwood, and the results showed that the NAA could significantly reduce the rooting Cycle, to improve the rooting rate and the quality of adventitious roots. Under the experimental conditions, the optimum NAA concentration was $600 \mathrm{mg} / \mathrm{L}$, which was 35.99\% higher than that of the control. The time of adventitious root was shortened by $9.48 \mathrm{~d}$ and the root length increased by $1.18 \mathrm{~cm}$, the number of rooting increased by 17.18. This is consistent with previous studies, and higher concentrations of rooting agents will affect the rooting time and rooting quality of cuttings [13-15].

(POD), polyphenol oxidase (PPO), superoxide dismutase (SOD) and indole acetic acid oxidase (IAAO) were all closely related to the rooting process Of the relationship, a direct impact on plant rooting characteristics. (POD), polyphenol oxidase (PPO), superoxide dismutase (SOD) and indole acetic acid oxidase (IAAO) were significantly changed by different NAA treatments. Oxidase activity, and in the process of rooting it is into a regular change. The activity of polyphenol oxidase (PPO), superoxide dismutase (SOD) and indole acetic acid oxidase (IAAO) increased gradually after cutting, reached the peak at the root stage and then decreased gradually. The activity of polyphenol oxidase (PPO) increased first and then decreased, which indicated that the formation and differentiation of callus and the induction of adventitious roots were needed in the process of rooting of hardwood cuttings Phenol oxidase (PPO), while in the adventitious root elongation stage requires low activity of polyphenol oxidase (PPO). It has also been shown that PPO activity is closely related to plant resistance [16-17], PPO activity increases when plants are exacerbated by adverse reactions, and PPO activity begins to decrease at the later stage of cutting, which may be due to adventitious roots Elongation, the number of cells increased, PPO involved in the synthesis of cell walls and lignin [18]. The growth of the oxygen free radicals or superoxide anions increases, which increases the cell membrane permeability and the superoxide dismutase (SOD) on the membrane lipid peroxidation. The role of protection, so after cutting to the root formation period of this stage of activity gradually increased, and reached the maximum. The activity of indoleacetic acid oxidase (IAAO) increased first and then decreased, because the low activity of IAAO could reduce the consumption of IAA and increase the IAA content in the cuttings, and the high concentration of IAA could promote the cutting Adventitious root formation and growth. This trend is not consistent with previous studies on other species. It is presumed that this is the result of the combination of hormonal species and relative proportions, the presence or absence of rooting inhibitors, the nutrient content in the cuttings, and other factors, but this speculation remains to be further studied. The activity of peroxidase (POD) increased gradually after cutting, reached its peak 
at the root stage, then gradually decreased, then increased gradually, reached another peak at the root growth stage, and then with the elongation of the root Gradually declining, showing a "rise drop - rise - decline" bimodal trend, which is consistent with previous research results [19-21].

NAA changes the activity of enzymes such as peroxidase (POD), polyphenol oxidase (PPO), superoxide dismutase (SOD) and indoleacetic acid oxidase (IAAO), etc., thereby promoting cell dedifferentiation Callus. In this study, the physiological response mechanism of rooting of hardwood cuttings was revealed from the changes of related oxidase in rooting process. However, due to the factors affecting the rooting of plant cuttings, only the contents of nutrient, the level of endogenous hormones And the rooting-related indicators of the law of change to be studied in order to more accurately explain the red sandalwood hardwood cutting rooting mechanism.

\section{Acknowledgements}

Fund Project: Hainan Provincial Natural Science Foundation (Grant No. 317181); Hainan Provincial Higher Education Research Project (No: Hnky2016ZD-13).

\section{References}

[1] Wang Weibin. China's mahogany tree species and its sustainable development countermeasures[J]. Fujian Forestry Science and Technology, 2003, 30 (4): 108 - 111.

[2] Wu Guoxin, Wang Linghui, Yu Jianmei, et al.Study on annual growth rhythm of red sandalwood seedlings [J]. Journal of Zhejiang Forestry Science and Technology, 2010, 30 (3): 56 60 .

[3] Shi Fujun, Yu Jianmei, Wang Linghui.Study on Cutting Propagation Technique of Malus hupehensis [J] .Guangdong Agricultural Sciences, 2011, 38 (1): 50 - 52.

[4] Hao Zaibin, Cang crystal, Xu Zhong. Plant Physiology Experiment [M]. Heilongjiang: Harbin Institute of Technology Press, 2004: 87-88.

[5] Li He Sheng. Plant physiological and biochemical experimental principles and technology [M]. Beijing: Higher Education Press, 1999: 35-37.

[6] Zhang Zhiliang, Qu Weijing. Plant Physiology Experimental Guidance [M]. Beijing: Higher Education Press, 2004: 188 - 189.

[7] Gaspar T, Kevers C, Hausman J F, et al. Practical uses of peroxidase activity as a predictive marker of rooting performance of micropropagated shoots[J]. Agronomie, 1992, 12(10): 757 - 765.

[8] Nordström A C, Eliasson L. Levels of endogenous indole-3-acetic acid and indole-3-acetylaspartic acid during adventitious root formation in pea cuttings[J]. Physiologia Plantarum, 1991, 82(4): 599 - 605.

[9] Haissig B E. Influences of auxins and auxin synergists on adventitious root primordium initiation and development[J]. New Zealand Journal Of Forestry Science, 1974, 4(2): 311 - 323.

[10] Al Barazi Z,Schwabe W W. The possible involvement of polyphenoloxidase and the austin-oxidase system in root formation and development in cuttings of Pistacia vera[J] Journal of horticultural science, 1984, 59(3): 453 - 461.

[11] Van C W, Inzé D, Van M M. The regulation and function of tobacco superoxide dismutases[J]. Free Radical Biology and Medicine, 1997, 23(3): 515 - 520. 